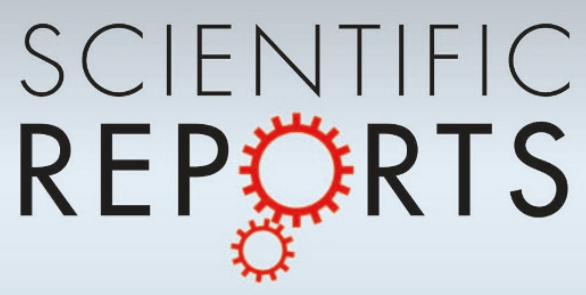

OPEN

SUBJECT AREAS:

OPTICAL SPECTROSCOPY

SURFACES, INTERFACES AND

THIN FILMS

ELECTRONIC PROPERTIES AND MATERIALS

Received

19 November 2014

Accepted

23 February 2015

Published

17 March 2015

Correspondence and requests for materials should be addressed to E.E.M.C. (elbertchia@ ntu.edu.sg)

\title{
New Insights into the Diverse Electronic Phases of a Novel Vanadium Dioxide
} Polymorph: A Terahertz Spectroscopy
Study

James Lourembam' ', Amar Srivastava ${ }^{2,3}$, Chan La-o-vorakiat ${ }^{1}$, H. Rotella ${ }^{2,4}$, T. Venkatesan ${ }^{2,3,5}$ \& Elbert E. M. Chia ${ }^{1}$

\begin{abstract}
'Division of Physics and Applied Physics, School of Physical and Mathematical Sciences, Nanyang Technological University, Singapore 637371, Singapore, ' $\mathrm{NUSNNI-Nanocore,} \mathrm{National} \mathrm{University} \mathrm{of} \mathrm{Singapore,} \mathrm{Singapore} 117411$, Singapore, ${ }^{3}$ Department of Physics, National University of Singapore, Singapore 117542 , Singapore, ${ }^{4}$ Singapore Synchrotron Light Source, National University of Singapore, 5 Research Link, Singapore 117603, 5 Department of Electrical and Computer Engineering, National University of Singapore, Singapore 117576, Singapore.
\end{abstract}

A remarkable feature of vanadium dioxide is that it can be synthesized in a number of polymorphs. The conductivity mechanism in the metastable layered polymorph $\mathrm{VO}_{2}(B)$ thin films has been investigated by terahertz time-domain spectroscopy (THz-TDS). In $\mathrm{VO}_{2}(B)$, a critical temperature of $240 \mathrm{~K}$ marks the appearance of a non-zero Drude term in the observed complex conductivity, indicating the evolution from a pure insulating state towards a metallic state. In contrast, the $\mathrm{THz}$ conductivity of the well-known $\mathrm{VO}_{2}(M 1)$ is well fitted only by a modification of the Drude model to include backscattering. We also identified two different $\mathrm{THz}$ conductivity regimes separated by temperature in these two polymorphs. The electronic phase diagram is constructed, revealing that the width and onset of the metal-insulator transition in the $B$ phase develop differently from the $M 1$ phase.

M ulti-polymorphic materials - compounds that can assume numerous, different crystal symmetries with the same chemical composition, show great promise as future electronic materials. This is because their structural diversity can give rise to a variety of electrical and optical responses that can be tuned for technological applications such as optical switches, batteries, solar cells, optical filters, spintronic devices, memory devices etc ${ }^{1-4}$. Vanadium dioxide is one of the most popular polymorphic materials that can assume several crystallographic structures ${ }^{5-7}$. Amongst the several stable and metastable $\mathrm{VO}_{2}$ polymorphs, monoclinic $\mathrm{VO}_{2}(B)$ and monoclinic $\mathrm{VO}_{2}(M 1)$ are particularly interesting, as these materials display more than thousand-fold changes in conductivity with temperature ${ }^{8,9}$. Understanding polymorphism in vanadium dioxide has tremendous practical importance in the design and control of electro-optic materials for future technologies.

The metastable monoclinic $\mathrm{VO}_{2}(B)$ adopts a structure derived from $\mathrm{V}_{2} \mathrm{O}_{5}$ and belongs to the space group $C 2 / \mathrm{m}$ (12) compared to the well-known $\mathrm{VO}_{2}(M 1)$, which takes up the space group $P 2_{1} / c(14)^{10-12}$. The crystal structure of $\mathrm{VO}_{2}(\mathrm{~B})$ is illustrated in Figure 1(a). A salient structural difference between the monoclinic phases in $\mathrm{VO}_{2}(B)$ and $\mathrm{VO}_{2}(M 1)$ is that $\mathrm{VO}_{2}(B)$ has a smaller $\beta$ inter-axial angle ${ }^{11}\left(\beta_{v o_{2}(B)}=106.6^{\circ}\right.$ and $\left.\beta_{v o_{2}(M 1)}=122.6^{\circ}\right)$ which may play an important role in the formation of defects and impurities in epitaxial growth of films. While metalinsulator transition (MIT) in $\mathrm{VO}_{2}(M 1)$ is associated with a corresponding reversible structural phase transition from a room temperature monoclinic to a high temperature tetragonal phase ${ }^{9,13-16}$, currently there is little understanding of the nature of the semimetal-to-insulator transition in $\mathrm{VO}_{2}(B)$ which remains monoclinic with $\mathrm{MIT}^{10}$. It is also interesting to compare these polymorphs in context of $\mathrm{V}-\mathrm{V}$ pairing mechanism, which strongly influences the conductivity pathways. $\mathrm{VO}_{2}(M 1)$ undergoes $\mathrm{V}-\mathrm{V}$ dimerization in the monoclinic insulating phase with very short $\mathrm{V}$-V bonds $(\sim 2.65 \AA)$ creating a large dimer-to-dimer distance $(\sim 3.12 \AA)^{14}$. This alternate long and short $\mathrm{V}-\mathrm{V}$ separation inhibits conductivity. But when $\mathrm{VO}_{2}(M 1)$ undergoes a phase transition to the rutile structure all the $\mathrm{V}-\mathrm{V}$ distances become the same $(\sim 2.87 \AA)$ and do not allow the formation of dimers ${ }^{14}$. In contrast, one doesn't see a significant change of V-V distance in the $B$ phase ${ }^{10}$. One could plainly see that, even though these $\mathrm{VO}_{2}$ polymorphs have the same chemical stoichiometry, their structures and electronic attributes 

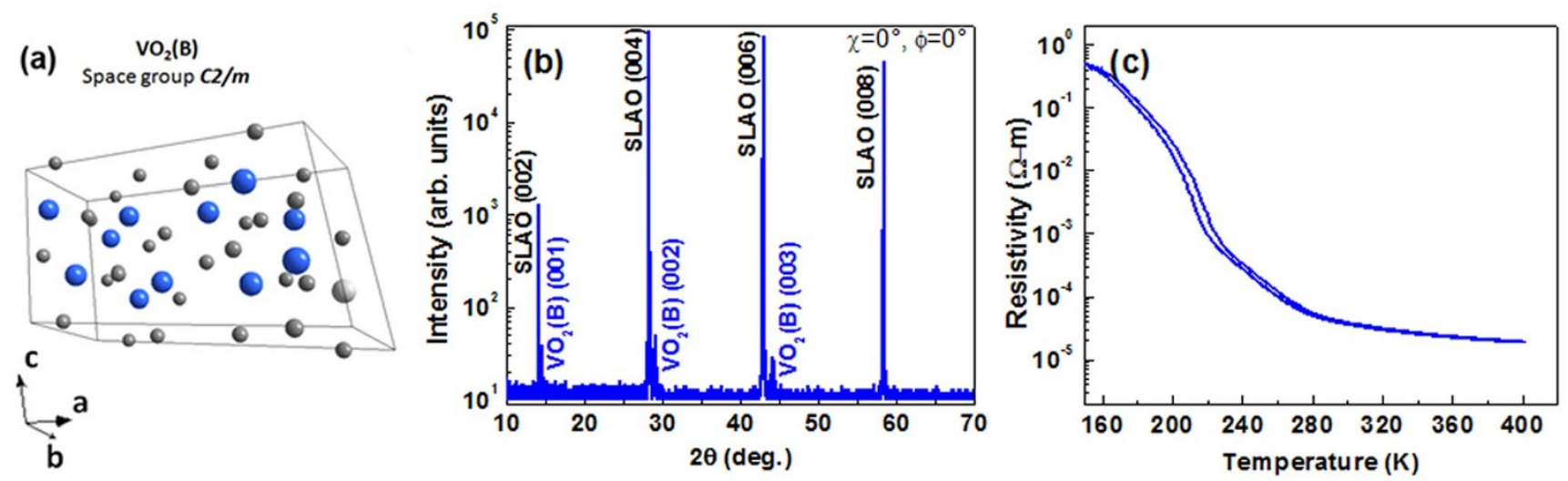

Figure $1 \mid$ (a) Crystal structure of the $\mathrm{VO}_{2}(B)$ polymorph. The vanadium atoms are represented by blue spheres and the oxygen atoms are represented by dark grey spheres.(b) XRD $\theta-2 \theta$ pattern for the $\mathrm{VO}_{2}(B)$ film epitaxially grown on (001) SLAO substrate. (c) Temperature dependent resistivity of $\mathrm{VO}_{2}(B)$ on SLAO measured using a traditional four point geometry.

are evidently distinct and a comparative study between these polymorphs is aptly motivated by an objective to get a better understanding on the correlation between MIT and structural phase transition. Furthermore, while a lot of progress has been made towards understanding the physics of metal-insulator transition in $\mathrm{VO}_{2}(M 1)$ by optical techniques ${ }^{17-28}$, no parallel efforts has been made so far to understand the mechanism in $\mathrm{VO}_{2}(B)$. From a practical applications point of view, one of the key attractions of $\mathrm{VO}_{2}(B)$ is that it is conducting at room temperature, compared to $\mathrm{VO}_{2}(M 1)$ which is insulating and may hold technological merits over $\mathrm{VO}_{2}(M 1)$ for devices at room temperature operation ${ }^{8}$.

The drastic changes in conductivity with temperature for these $\mathrm{VO}_{2}$ polymorphs are accompanied by a corresponding change in optical response, thereby making terahertz $(\mathrm{THz})$ spectroscopy a viable technique to probe the nature of electronic properties in these systems. There have been several reports where $\mathrm{THz}$ spectroscopy has been employed to measure conductivity in $\mathrm{VO}_{2}(M 1)$ polymorphs $s^{25,26,29-31}$. Although metal-insulator transition materials are generally characterized by standard four-point probe method, it provides information only about dc conductivities which can be distorted by surface defects due to electrode contacts ${ }^{32}$. On the other hand, $\mathrm{THz}$ spectroscopy is a contactless probe, providing frequency-dependent complex conductivity in the far-infrared region. Terahertz time-domain spectroscopy (THz-TDS) allows the amplitude and phase of the THz pulse to be obtained, without the need for Kramers-Kronig analysis in the extraction of complex conductivity ${ }^{33-35}$. Moreover, compared to traditional steadystate dc conductivity measurements which is limited to macroscopic conductivity paths, $\mathrm{THz}$ spectroscopy explores electron dynamics over nanometer length scales, possibly revealing conducting phases in this scale undetectable by long-range transport measurements ${ }^{29}$. Indeed, $\mathrm{THz}$ spectroscopy has been used to make comprehensive optical investigations in a host of materials rich in exotic conducting phases including graphene ${ }^{36,37}$, toplogical insulators ${ }^{34,38}$, superconductors ${ }^{39-41}$, oxide semiconductors $^{32,33}$, quantum-confined semiconductors ${ }^{42}$, and percolating systems ${ }^{43}$.

In this paper we report a systematic investigation of $\mathrm{THz}$ complex conductivities of 60-nm thick $\mathrm{VO}_{2}(B)$ thin films deposited on (001) $\mathrm{SrLaAlO}_{4}(\mathrm{SLAO})$ substrate. There has been no literature report on the complex conductivities of this system. In our operational $\mathrm{THz}$ frequency range (0.3-2.3 THz), there are no observable optical phonon resonances of $\mathrm{VO}_{2}(B)$, and hence it is possible to observe the conductivity response that arises solely from free carriers. These are then compared with that of $60-\mathrm{nm} \mathrm{VO}_{2}(M 1)$ thin films deposited on SLAO substrate. This study conclusively demonstrates clear signatures of different electronic orders in these two vanadium dioxide polymorphs.

\section{Results}

Sample growth, structural and transport characterization. The XRD spectra of $\mathrm{VO}_{2}(B)$ thin film on SLAO is shown in Figure 1(b). We clearly see that the XRD reflections of $\mathrm{VO}_{2}(B)$ are perfectly aligned with the substrate (azimuthal angle, $\chi=0$ ). This means that our film is highly oriented. Temperature-dependent electrical transport measurements are carried out in Physical Property Measurement system (Quantum Design) utilizing a four probe geometry. As shown in Figure $1(\mathrm{c})$, the resistivity of $\mathrm{VO}_{2}(B)$ films grown on SLAO substrate undergo four orders-of-magnitude change (from $2 \times 10^{-5} \Omega$-m to 0.35 $\Omega-\mathrm{m}$ ) in resistivity as the temperature changes from $400 \mathrm{~K}$ to $160 \mathrm{~K}$, which is consistent with a previous report on $\mathrm{VO}_{2}(B)$ nanorods ${ }^{8}$. There is a weak thermal hysteresis at low temperatures below $240 \mathrm{~K}$.

THz-TDS. Figure 2(a) shows time-domain signal of the main $\mathrm{THz}$ pulse $[E(t)]$ transmitted through the film and reference for $\mathrm{VO}_{2}(B)$ thin film. After the main pulse, a weaker pulse (etalon pulse) appears due to multiple reflections in the substrate. The main $\mathrm{THz}$ pulse and etalon pulse are well separated in the time domain, allowing easy truncation of the time-domain data to remove the etalon pulse. All our subsequent data analysis is done on the main pulse and removal of etalon pulses does not lead to any relevant loss of information. The lower panel of Figure 2(a) is the $\mathrm{THz}$ absorption at $295 \mathrm{~K}$ by plotting the difference in the electric field time domain signal between the sample and the reference $[\Delta E(t)]$. Fast Fourier Transform (FFT) is then performed on the time domain $\mathrm{THz}$ signals to obtain the amplitude and phase of the $\mathrm{THz}$ transmission spectra.

The transmittance, $T(\omega)$ of the $\mathrm{VO}_{2}$ film is defined as the ratio between complex electric field of the $\mathrm{THz}$ pulse from sample (film + substrate) $\tilde{E}_{s}(\omega)$ and reference (bare substrate) $\tilde{E}_{r}(\omega)$. In Figure $2(\mathrm{~b})$ the frequency-dependent and temperature-dependent transmittance amplitudes through the $\mathrm{VO}_{2}(B)$ film are shown. In the insulating state, at temperatures lower than the transition temperature, we find that the transmittance amplitude is close to unity. For $\mathrm{VO}_{2}(B)$ film, as the temperature of the sample approaches $\sim 240 \mathrm{~K}$, there is $\mathrm{THz}$ absorption and a clear trend of increasing absorption at higher temperatures. The spectral shape is almost frequency independent for all runs. The frequency dependent complex refractive index $\tilde{n}=n+i \kappa$ can be extracted from the experimental transmittance $T(\omega)$ by fitting the formula ${ }^{36,44}$

$$
T(\omega)=\frac{2 \tilde{n}\left(\tilde{n}_{s u b}+1\right) \exp \left[\frac{i \omega(\tilde{n}-1)}{c}\right] \exp \left[-\frac{i \omega \Delta L\left(\tilde{n}_{s u b}-1\right)}{c}\right]}{(1+\tilde{n})\left(\tilde{n}+\tilde{n}_{s u b}\right)+(\tilde{n}-1)\left(\tilde{n}_{s u b}-\tilde{n}\right) \exp \left[\frac{2 i \omega d \tilde{n}}{c}\right]}
$$

where $\tilde{n}$ and $\tilde{n}_{\text {sub }}$ are the complex refractive indices of $\mathrm{VO}_{2}$ film and SLAO substrate, respectively, $d$ is film thickness and $\Delta L$ is the thickness 
(a)

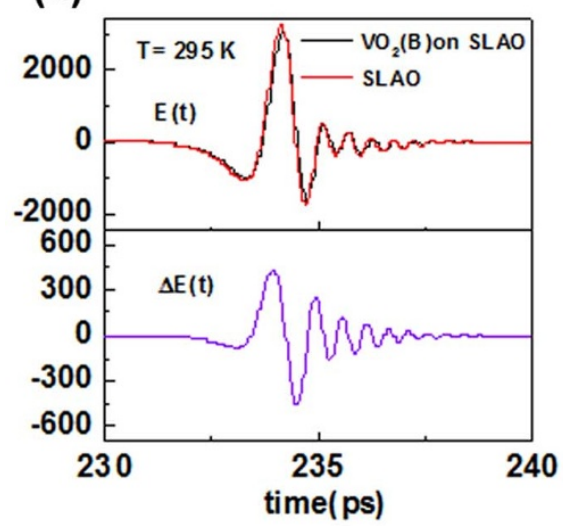

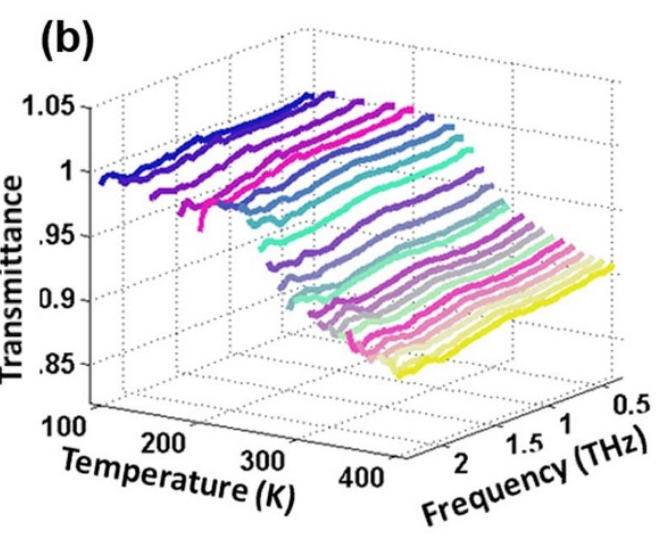

Figure $2 \mid$ (a) $\mathrm{THz}$ signals transmitted through $\mathrm{VO}_{2}(B)$ film deposited on SLAO substrate compared to the transmitted signal through bare substrate at $295 \mathrm{~K}$ shown along with monoclinic $\mathrm{VO}_{2}(B)$ cell. While the top panel describes the raw transmitted THz electric-field waveform $E(t)$ the bottom panel represents the corresponding difference in the THz waveform $\Delta E(t)$ between the sample and the reference. (b) Evolution of the transmission spectra with temperature and frequency of $\mathrm{VO}_{2}(B)$ thin film deposited on SLAO substrates during warming process.

difference between sample and reference substrates and $c$ is the speed of light in vacuum. This above equation takes into account the multiple internal reflections inside the film.

The extracted complex refractive index $\tilde{n}$ is then used to calculate the complex optical conductivity $\tilde{\sigma}=\sigma_{1}+i \sigma_{2}$ where $\sigma_{1}(\omega)=2 n \kappa \omega \varepsilon_{0}$ and $\sigma_{2}(\omega)=\left(\varepsilon_{\infty}-n^{2}+\kappa^{2}\right) \omega \varepsilon_{0}$ where $\varepsilon_{0}$ is the permittivity of free space and $\varepsilon_{\infty}$ is the high frequency dielectric constant. For $\mathrm{VO}_{2}(B), \varepsilon_{\infty}$ is an unknown quantity and is initially set to 1 . After the determination of complex conductivities, $\varepsilon_{\infty}$ will be used as a temperature-dependent fitting parameter in the conductivity models as discussed in the following sub-sections. On the other hand, we used $\varepsilon_{\infty}=9$ based on previous studies for determining conductivities in $\mathrm{VO}_{2}(M 1)^{45,46}$.

Complex conductivity of $\mathrm{VO}_{2}(\boldsymbol{B})$. The complex optical conductivities of the $\mathrm{VO}_{2}(B)$ films for temperatures ranging from $240 \mathrm{~K}$ to $400 \mathrm{~K}$ are shown in Figures 3(a) and (b) as a function of frequency during the

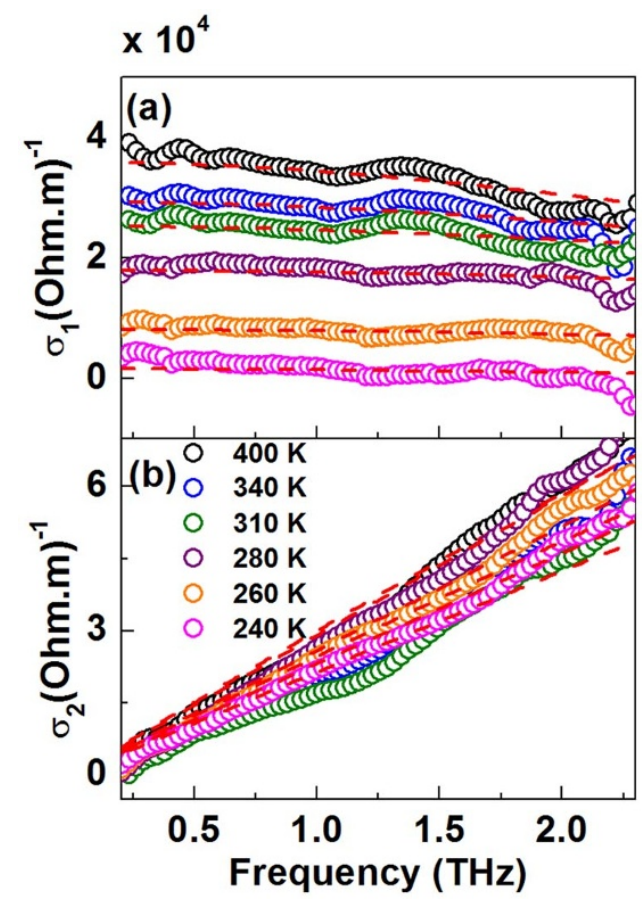

Figure 3 | (a) Real and (b) imaginary conductivities in the THz frequency range of $\mathrm{VO}_{2}(B)$ thin films grown on SLAO with fits to the Drude model (red dash lines) shown for temperatures ranging from 240 to $400 \mathrm{~K}$. warming process (open symbols). Below $240 \mathrm{~K}$, the transmission is $\sim 100 \%$, giving zero real conductivity, indicating that the films are clearly insulating. The onset of a positive $\sigma_{1}(\omega)$ marks the transition from an insulating regime to a conducting one. We clearly observe $\sigma_{1}(\omega)$ to increase with increasing temperature throughout our frequency range. Thermal excitation of electrons into the conduction band can account for this nature, that is, a Drude response where $\sigma_{1}(\omega)$ is a maximum at low frequency and decreases with increasing frequency, while $\sigma_{2}(\omega)$ are zero at low frequency and increases with increasing frequency.

According to the Drude model, the frequency dependent complex optical conductivity is given by

$$
\tilde{\sigma}=\frac{s_{0} \omega_{P}^{2}}{\gamma-i \omega}-i \varepsilon_{0} \omega\left(\varepsilon_{\infty}-1\right)
$$

where $\omega_{P}$ is the Drude plasma frequency, $\gamma$ is the free carrier scattering rate and $\varepsilon_{\infty}$ is the high frequency dielectric constant as defined earlier. Both $\sigma_{1}(\omega)$ and $\sigma_{2}(\omega)$ at each temperature measurement are simultaneously fitted to Equation 2, as shown by the dash red lines in Figures 3(a) and (b) respectively. The fits are reasonably good for all temperatures. The fittings results of the Drude parameters are shown in Figures 4(a) and (b). The simultaneous fitting of $\sigma_{1}(\omega)$ and $\sigma_{2}(\omega)$ to the Drude model greatly reduces the width of the error bars of the fitted $\gamma$ and $\omega_{P}$ even though $\sigma_{1}(\omega)$ is relatively featureless.

We observe a similar trend in both the plasma frequency and the scattering rate - both parameters increases as we increase the temperature up to $\sim 280 \mathrm{~K}$, and tends to saturate beyond $280 \mathrm{~K}$. This common trend in $\omega_{P}$ and $\gamma$ describe two different temperature-dependent conductivity mechanisms. To address this issue and to make a distinction, we describe the temperature regions $240 \mathrm{~K} \leq T \leq 280 \mathrm{~K}$ and $280 \mathrm{~K}<T \leq 400 \mathrm{~K}$ as conductivity regimes I and II respectively.

In regime $\mathrm{I}, \omega_{P} / 2 \pi$ rises from $(8 \pm 1) \mathrm{THz}$ to $(49 \pm 8) \mathrm{THz}$, while in regime II this parameter has a mean value of $\sim(54 \pm 5) \mathrm{THz}$. The plasma frequency is closely related to the number of charge carriers $(N)$ in the system via $\omega_{P}^{2}=N e^{2} / m^{*} \varepsilon_{0}$ where $m^{*}$ is the effective mass. The scattering rate, $(\gamma)$ is associated with the mobility of the carriers, $(\mu)$ via the expression $\gamma=e /\left(m^{*} \mu\right)$. Without the knowledge of the carriers' effective mass, accurate values of $N$ and $\mu$ in $\mathrm{VO}_{2}(B)$ cannot be determined. In the later sub-sections, more discussions regarding the nature of $m^{*}$ will be presented.

The Boltzmann dc conductivity $\sigma_{0}$ can be obtained from the expression

$$
\sigma_{0}=\frac{\varepsilon_{0} \omega_{P}^{2}}{\gamma}
$$




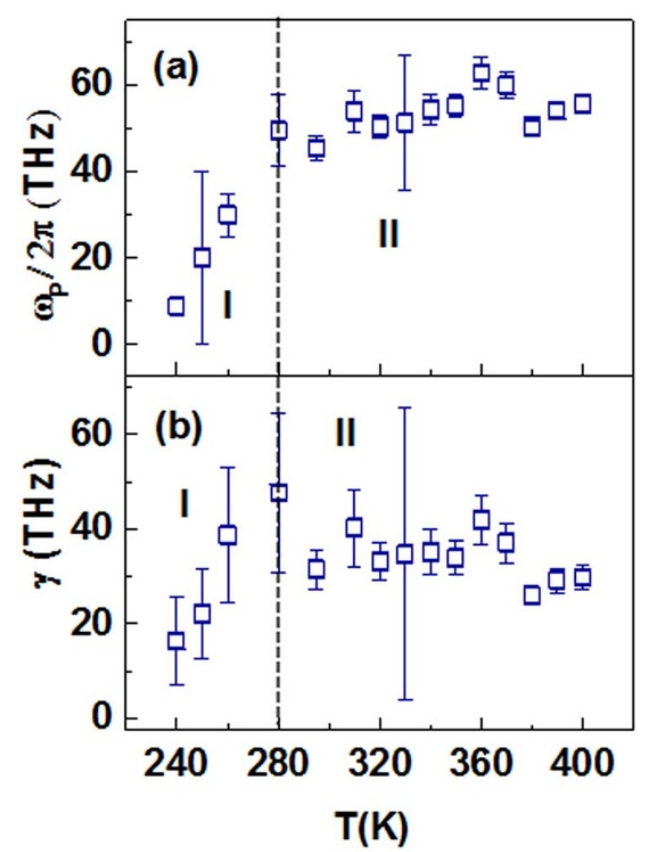

Figure $4 \mid$ Temperature dependence of (a) the plasma frequency and (b) the scattering rate, for $T \geq 240 \mathrm{~K}$ as estimated from the Drude fitting. The dashed lines are guide to the eye demarcating the two different electronic orders.

Figure 5(a) shows the calculated dc conductivity using Equation 3. The plot shows $\sigma_{0}$ increasing with increasing temperatures, but the rate of change is significantly smaller at higher frequencies. We recall that while $\omega_{P}$ and $\gamma$ start saturating at $280 \mathrm{~K}, \sigma_{0}$ does not. However, for $T>280 \mathrm{~K}$ and $T \leq 280 \mathrm{~K}, \sigma_{0}$ changes with different slopes and hence can be correlated with our description of conductivity regimes I and II. If we compare, our estimated dc conductivity from the $\mathrm{THz}$ measurements are consistent with the electrical transport measurements shown in Figure 1(c). Figure 5(b) shows the logarithmic conductivity, $\ln (\sigma)$ plotted against reciprocal temperature, 1000/T. The activation energy, $E_{a}$ can be estimated from the thermal activation model of $\mathrm{dc}$ conductivity which has the following temperature dependence

$$
\ln \sigma=A-\frac{E_{a}}{k_{B} T}
$$

where $k_{\mathrm{B}}$ is the Boltzmann constant. The linear fittings of the conductivity regimes with the activation model show a good agreement. Fits to Equation 4 yields $E_{a}$ (regime I) $=(260 \pm 30) \mathrm{meV}$, and $E_{a}$ (regime II $)=(47 \pm 3) \mathrm{meV}$. This significant difference in activation energies supports our claim that there are two different temperature dependent conductivity mechanisms in $\mathrm{VO}_{2}(B)$. In regime $\mathrm{I}$, the conductivity mechanism is poor with larger activation energy, while in regime II, the conductivity is better with smaller activation energy. The presence of small activation energy in conductivity regime II shows that even at high temperatures it has not achieved a fully metallic state. The features of metal-insulator transition in $\mathrm{VO}_{2}(B)$ can be understood as follows: (i) Temperatures below $200 \mathrm{~K}$ can be identified as a pure insulating state displaying $100 \%$ transmission in the insulating state, (ii) conducting state I is an intermediate electronic phase transition characterized by strong dependence of Drude parameters with temperature, (iii) conducting state II describes the phase after the transition is completed. The boundaries of various electronic phases driven by temperature are thus more clearly defined in the THz-TDS study compared to the electrical transport measurements where the change in resistivity is gradual and not well discernible.
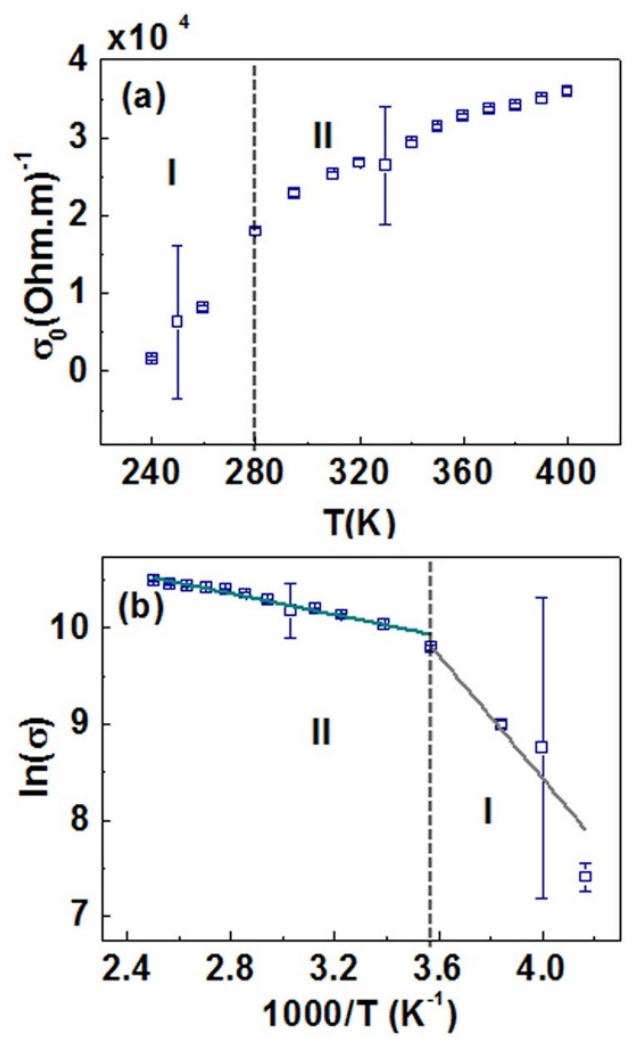

Figure $5 \mid$ (a) Temperature dependence of dc conductivity of $\mathrm{VO}_{2}(B)$ films on SLAO as estimated from Equation 3. (b) Logarithmic plot of conductivity $\ln (\sigma)$ vs reciprocal temperature 1000/T shown alongside linear fitting and the conductivity regimes I and II.

Comparison with $\mathrm{VO}_{2}(\mathbf{M 1})$. The $\mathrm{THz}$ conductivity spectra of 60 -nm thick $\mathrm{VO}_{2}(M 1)$ film grown on SLAO are shown in Figure 6 plotted as the temperature of the system is gradually increased from $343 \mathrm{~K}$ to $400 \mathrm{~K}$. In contrast to $\mathrm{VO}_{2}(B)$, throughout this temperature range, $\sigma_{1}(\omega)$ increases with increasing frequency, while $\sigma_{2}(\omega)$ starts at zero at low frequencies, becomes negative, then crosses over to positive with increasing frequency. This is consistent with the previous reported $\mathrm{THz}$ studies of $\mathrm{VO}_{2}(M 1)$ films ${ }^{25,26,30}$.

In classical $\mathrm{VO}_{2}(M 1)$ systems it has been reported that the complex conductivities can be effectively modelled by Drude-Smith theory, which is a generalisation of the Drude model proposed by Smith, in order to account for the conductivity suppression due to charge localization $^{33,43,47,48}$. In this polymorph, it is widely considered that the transition from a highly transparent insulating phase to an absorbing metallic phase occurs through a percolating network ${ }^{29,46,49}$. Near the threshold of this transition, phase separation and domain formation dictate the electronic pathways. The frequency-dependent optical conductivity in the Drude-Smith model is given by ${ }^{47}$

$$
\tilde{\sigma}=\frac{\varepsilon_{0} \omega_{P}^{2}}{\gamma-i \omega}\left[1+\sum j \frac{c_{j} \gamma}{\gamma-i \omega}\right]-i \varepsilon_{0} \omega\left(\varepsilon_{\infty}-1\right)
$$

The additional terms described by $c_{j}$ represents the fraction of the carrier's initial velocity retained after $j$ number of scattering events ${ }^{47}$. In various systems such as poor conductors, metal-insulator transition materials and various nanomaterials where the Drude-Smith model is successfully applied, only the first scattering term is considered (i.e. $j=1)^{30,48,50-52}$. In this scenario, carrier transport transitions from ballistic to diffusive after one scattering event, leading to complete momentum randomization ${ }^{47}$. In our fitting analysis also we will only consider the first $c_{j}$ term and in this case we take $c_{1}=c$. The parameter $c$ can take up any values anywhere between 0 (free Drude 


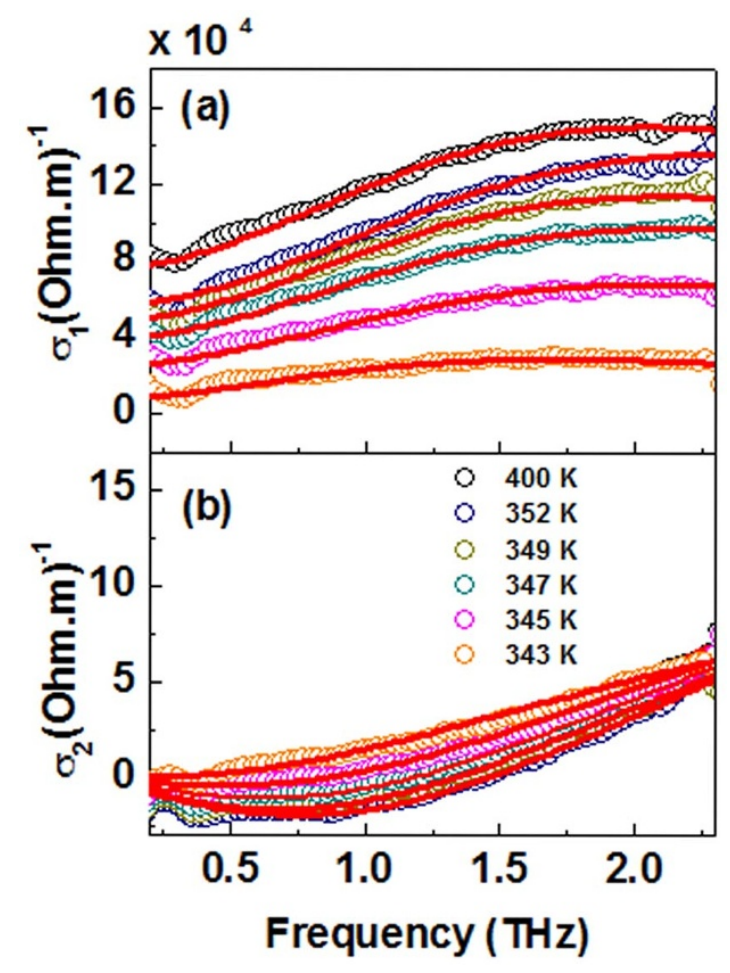

Figure 6 | (a) Real and (b) imaginary conductivities of $\mathrm{VO}_{2}(M 1)$ films on SLAO with fits to the Drude-Smith model (sold red lines) for six selected temperature points in the range $343 \mathrm{~K}-400 \mathrm{~K}$ during the warming process.

carrier conduction) and -1 (full carrier backscattering) ${ }^{43}$. There have been other reports of using effective medium theory (EMT) models to fit $\mathrm{THz}$ conductivities of $\mathrm{VO}_{2}(M 1)$ with varying degrees of success $^{29,31}$. Our attempts to model the optical conductivities using the effective medium models gave inconsistent results (Supplementary Information). On the other hand, as shown by the fitting lines in the Figures 6 (a) and (b), the Drude-Smith formalism given by Equation (5) when $j=1$ gives excellent fits to our measured conductivities of $\sigma_{1}(\omega)$ and $\sigma_{2}(\omega)$. We could see that even at high temperatures the conductivity does not follow an ideal Drude behaviour which indicates that the nanogranular boundaries persist even after the transition. In highly granular systems, the Drude-Smith is much more effective in describing the effective conductivity rather than a conventional EMT models because it can describe effective conductivities of systems where grain boundaries persist even in large conducting fractions ${ }^{30,43}$. The advantage of Drude-Smith over EMT models in this scenario has been discussed by T. L. Cocker et.al ${ }^{30}$.

Simultaneous complex fitting of optical conductivities gives the information about the parameters $\omega_{P}, \gamma$ and $c$. Figure 7 summarizes the $\omega_{P}, \gamma$ parameters obtained from the fitting procedure as well as the $\mathrm{dc}$ conductivity $\sigma_{0}$ which is given by the equation, $\sigma_{0}=$ $\frac{\varepsilon_{0} \omega_{P}^{2}(1+c)}{\gamma}$. The Drude-Smith parameters $\omega_{P}$ and $\gamma$ increase with increasing temperatures in the temperature range $343 \mathrm{~K}-349 \mathrm{~K}$; above $349 \mathrm{~K}$ they remain largely temperature-independent. This characteristic is similar to the temperature dependence of Drude parameters in $\mathrm{VO}_{2}(B)$. In the temperature range $352 \mathrm{~K}-400 \mathrm{~K}$, the mean value of $\omega_{P} / 2 \pi$ is $\sim(110 \pm 4) \mathrm{THz}$. The fitting parameter $c$ lies between -0.88 and -0.75 which indicates significant carrier backscattering - an attribute of carrier localization, resulting in significant suppression of low-frequency conductivities. The temperature dependence of the fitted values of $c$ supports the percolation picture in $\mathrm{VO}_{2}(M 1)^{43}$.

We find that similar to $\omega_{P}$ and $\gamma, \sigma_{0}$ increases with increasing temperatures till $349 \mathrm{~K}$; at higher temperatures, it attains a mean saturating value of $(6.5 \pm 0.3) \times 10^{4}(\mathrm{Ohm} \cdot \mathrm{m})^{-1}$. Analogous to $\mathrm{VO}_{2}(B)$, we can define the temperature range of $343 \mathrm{~K}-349 \mathrm{~K}$ as conductivity regime I and $352 \mathrm{~K}-400 \mathrm{~K}$ as conductivity regime II. However in $\mathrm{VO}_{2}(M 1), \sigma_{0}$ remains largely temperature independent in conductivity regime II, while in $\mathrm{VO}_{2}(B)$ there is a weak temperature dependence in this regime. Figure 7(d) shows the linear fitting plot of logarithmic conductivity, $\ln (\sigma)$ versus reciprocal temperature, $1000 / T$. Similar to our previous analysis of $\mathrm{VO}_{2}(B)$, we can determine the activation energy of $\mathrm{VO}_{2}(M 1)$ from the slope of the linear relation. For $\mathrm{VO}_{2}(M 1)$, in the low-temperature regime I, the activation energy $E_{a}=(2200 \pm 500) \mathrm{meV}$ while in the high-temperature state II, $E_{a}=(20 \pm 8) \mathrm{meV}$. The difference between the activation energies in regions I and II is much larger in $\mathrm{VO}_{2}(M 1)$ than in $\mathrm{VO}_{2}(B)$ which is consistent with our $\omega_{P}$ and $\sigma_{0}$ results where the quantitative growth of these values as the system goes from regime I to II is much larger in $\mathrm{VO}_{2}(M 1)$ compared to $\mathrm{VO}_{2}(B)$. Also, while the activation
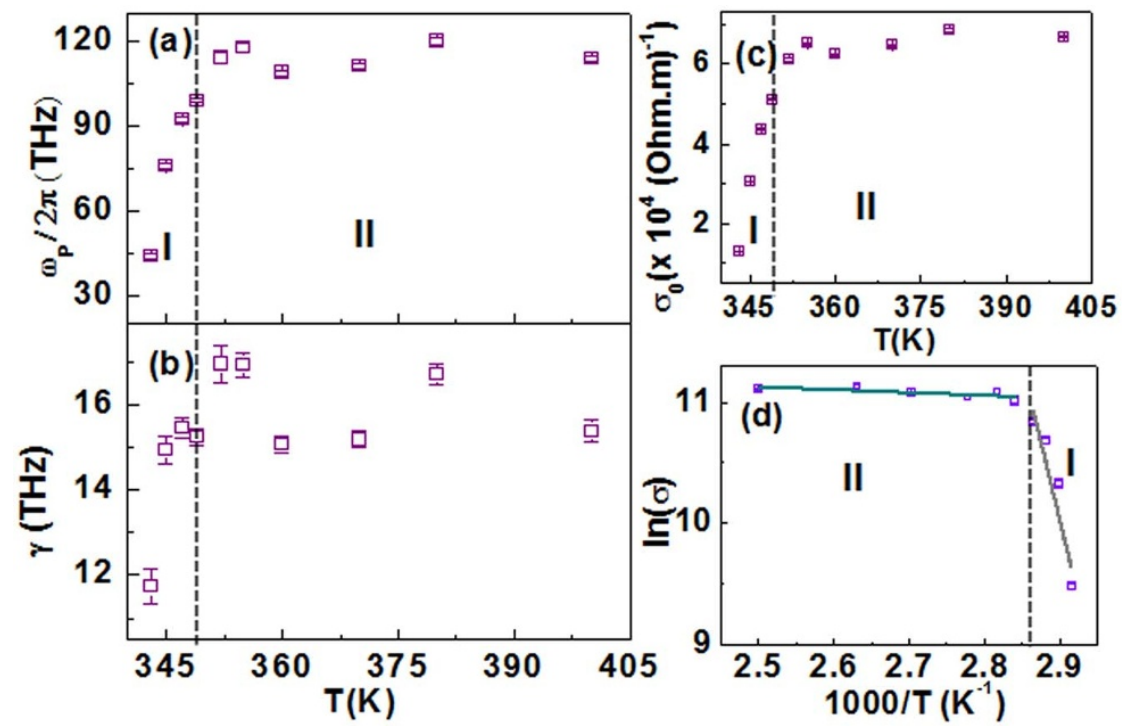

Figure $7 \mid$ Temperature dependence of Drude-Smith fitting parameters: (a) the plasma frequency, $\omega_{P} / 2 \pi$ (b) the scattering rate, $\gamma$ for $T \geq 343 \mathrm{~K}$ and (c) dc conductivity as estimated from Equation 3 for $\mathrm{VO}_{2}(M 1)$ films grown on SLAO during the warming cycle. The dashed lines separates the conductivity regimes I and II. (d) Logarithmic conductivity, $\ln (\sigma)$ dependence on reciprocal temperature $1000 / T$ is shown and fitted with a linear relation. 
energies in regime II are similar in these $\mathrm{VO}_{2}$ polymorphs, $E_{a}$ of $\mathrm{VO}_{2}(M 1)$ is an order of magnitude larger than that of $\mathrm{VO}_{2}(B)$ in regime $\mathrm{I}$. This means that in regime $\mathrm{I}$, the conductivity slope is steeper in $\mathrm{VO}_{2}(M 1)$ and concomitantly we found a sharper rise of $\omega_{P}$ and $\gamma$ in $\mathrm{VO}_{2}(M 1)$ in regime $\mathrm{I}$.

According to M. M. Qazilbash et. al. ${ }^{22}$, a significant enhancement of the carrier effective mass, $m^{*}$ (as large as $5 m_{e}$, where $m_{e}$ is mass of an electron) is found in the infrared spectra, especially in the intermediary phase transition state which is analogous to our conductivity regime I. After the transition is completed (at $T \sim 350 \mathrm{~K}$ ), $m^{*}$ is close to $2 m_{e}$ which is the value reported by other experiments ${ }^{46}$. Considering $m^{*}=2 m_{e}$, at temperatures just above the transition $(T=352 \mathrm{~K})$, we obtain the carrier density to be $\sim 3.6 \times 10^{20} \mathrm{~cm}^{-3}$ from the plasma frequency. In the case of Drude-Smith model, the relationship between $\gamma$ and $\mu$ is modified as, $\gamma=\frac{(1+c) e}{m^{*} \mu}$. This relationship gives $\mu=11.35 \mathrm{~cm}^{2} /(\mathrm{V} \cdot \mathrm{s})$ at $T=352 \mathrm{~K}$ in $\mathrm{VO}_{2}(M 1)$. Previous $\mathrm{THz}$-TDS experiments on $\mathrm{VO}_{2}(M 1)$ reported similar values of carrier density and mobility ${ }^{30,31}$. Hall effect measurements reported similar values of carrier density at this temperature but the reported mobility is lower, which is probable as $\mathrm{THz}$ study does not face the challenges in Hall measurements due to low-mobility ${ }^{53}$. The fundamental difference between these two techniques is that while Hall measurement is meant for DC conductivity measurements $\mathrm{THz}$-TDS is an AC measurement method. Hall measurement being a contact method requires the charge carriers to travel substantial distance across the biased electrodes but on the other hand THz-TDS captures the conductivity traversed in sub-pico second timescales resulting in 'conductivity snap-shots' ${ }^{42}$. As a consequence, $\mathrm{THz}$ TDS can give conductivity information before the carriers are trapped or recombined and as such it is quite reasonable to expect that the $\mathrm{THz}$ mobility would be higher than the Hall mobility. We can also compare the carrier density and the mobility parameters of $\mathrm{VO}_{2}(M 1)$ with that of $\mathrm{VO}_{2}(B)$. By assuming $m^{*}=2 m_{e}$ at $T=295 \mathrm{~K}$ for $\mathrm{VO}_{2}(B)$, which is just after the transition, the calculated values are $N \approx 6.4 \times 10^{19} \mathrm{~cm}^{-3}$ and $\mu \approx 21.9 \mathrm{~cm}^{2} /(\mathrm{V} \cdot \mathrm{s})$.

It has been reported that the first-order phase transition in $\mathrm{VO}_{2}(M 1)$ takes place via percolation where metallic domains embedded in a matrix of insulating phase starts nucleating sporadically as temperature is increased. When the temperature reaches the metalinsulator transition temperature $T_{M I}$, the domains become well connected to form a percolating path in this inhomogeneous composite medium. The Drude-Smith model has been applied to various percolating systems where inhomogeneity in conductivity contributes of

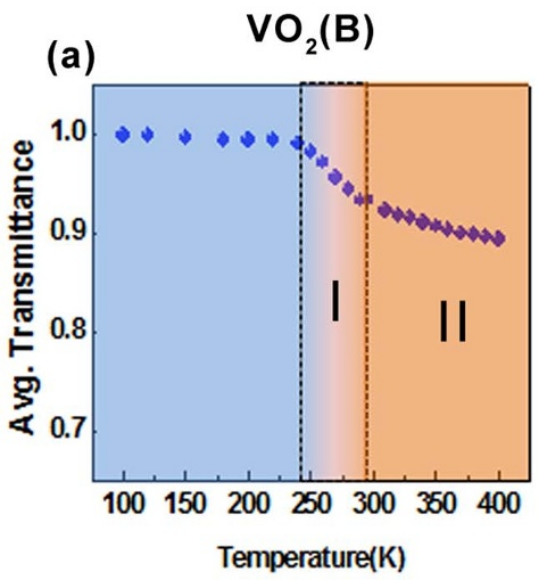

a non-zero backscattering parameter $c$. On the other hand $\mathrm{VO}_{2}(B)$ can be described by a simple Drude model, although it is a poor conductor in comparison to $\mathrm{VO}_{2}(R)$. The fact that $\mathrm{VO}_{2}(R)$ cannot have an ideal Drude behavior can be explained by its granular origin, intrinsic inhomogeneity. In $\mathrm{VO}_{2}(B)$ we cannot see evidence of inhomogeneous phase separation or percolating transport from the $\mathrm{THz}$ study and thus shows a good Drude fit. The explanation for this difference between the polymorphs is two-fold - first, $\mathrm{VO}_{2}(B)$ is a more uniform film in the context of epitaxial growth with lesser roughness and hence no backscattering. $\mathrm{VO}_{2}(M 1)$ on the other hand, has a more grainy morphology due to the larger $\beta$ inter-axial angle (Supplementary Information). Second, the activation energy is larger in $\mathrm{VO}_{2}(B)$ in the conducting state and so the number of thermally activated carriers are smaller.

Furthermore, the narrow intermediate state of $\mathrm{VO}_{2}(M 1)$, which falls in our conductivity regime $\mathrm{I}$, is a strongly correlated phase marked by enhanced mass and an optical pseudo-gap before crossing over to a rutile metallic state as indicated by several optical measurements including infrared spectroscopy, ellipsometry and femtosecond pump-probe measurements $\mathrm{s}^{22,23,49,54}$. It is also possible that in the intermediate state of $\mathrm{VO}_{2}(B)$, electronic correlations may play a significant role in this intermediate transition state. The rich electronic phase diagram of these vanadium dioxide polymorphs can be summarized from our $\mathrm{THz}$ transmission spectra as illustrated in Figure 8 .

\section{Discussion}

In conclusion, we have used $\mathrm{THz}$ spectroscopy to measure the temperature-dependent complex optical conductivity of vanadium dioxide polymorphs. In our operational frequency range $(0.3-2.3) \mathrm{THz}$, $\mathrm{VO}_{2}(B)$ exhibits no phonon modes and the onset of Drude conductivity behaviour arrives for temperatures higher than $240 \mathrm{~K}$. On the other hand, in $\mathrm{VO}_{2}(M 1)$ the $\mathrm{THz}$ conductivity can be better described by the Drude-Smith model. Key parameters of carrier dynamics (i) plasma frequency $\left(\omega_{P}\right)$ and (ii) scattering rate $(\gamma)$ are obtained by fitting to the Drude (in $\mathrm{VO}_{2}(B)$ ) and Drude-Smith in $\left(\mathrm{VO}_{2}(M 1)\right)$ models. Interestingly, our $\mathrm{THz}$ analysis revealed that like in $\mathrm{VO}_{2}(M 1), \mathrm{VO}_{2}(B)$ also transforms from an insulating system to a conducting system but is mediated by a much broader intermediate state with the transition onset much closer to room temperature making it more suitable than the widely studied $\mathrm{VO}_{2}(M 1)$ for optoelectronic devices operating at room temperature. Our study also demonstrates that these polymorphs can also be used as temperature-controlled $\mathrm{THz}$ frequency modulators. While the sharp phase

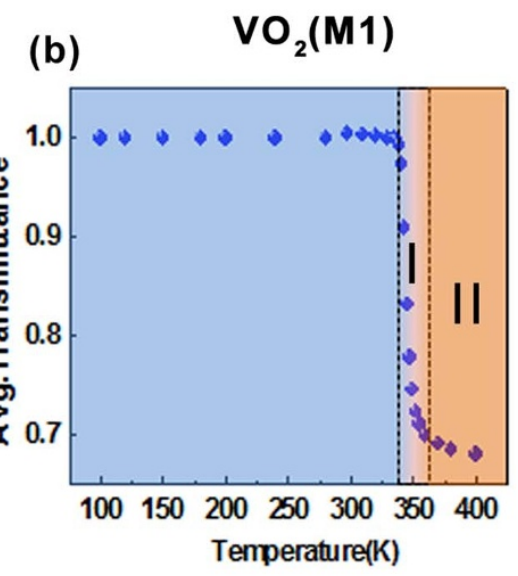

Figure 8 $\mid$ Normalized and frequency-averaged $\mathrm{THz}$ transmission of $(\mathrm{a}) \mathrm{VO}_{2}(B)$ and $(\mathrm{b}) \mathrm{VO}_{2}(M 1)$ thin films as a function of temperature during the warming process. These values are normalised to the transmission at $100 \mathrm{~K}$. The blue colour regions represent the insulating phases while the pure conducting phases are coloured orange. The two dotted lines mark the intermediate transition state. The conductivity regimes I and II as described in the previous discussions are also labelled. 
transition in $\mathrm{VO}_{2}(M 1)$ is suitable for digital-like $\mathrm{THz}$ modulation, the broad phase transition in $\mathrm{VO}_{2}(B)$ offers analog-like continuous modulation of $\mathrm{THz}$ spectra very similar to $\mathrm{W}$-doped $\mathrm{VO}_{2}(M 1)^{55}$. $\mathrm{THz}$ conductivity study is a fascinating subject of research from viewpoints of phase transitions in these systems. We show that THz spectroscopy is an excellent method of studying polymorphism in materials.

\section{Methods}

Sample growth. Pulsed Laser Deposition is used to fabricate the various $\mathrm{VO}_{2}$ polymorph thin films by ablating a commercial Vanadium single crystal (100) orientated target on the SLAO (001) substrate. The growth temperature is $500^{\circ} \mathrm{C}$ and the oxygen pressure is varied from $5 \times 10^{-3}$ to $7 \times 10^{-3}$ Torr. By systematic control of oxygen growth pressure and pulse laser frequency, the various phases of $\mathrm{VO}_{2}$ are stabilized. Detailed growth conditions study has been reported in a previous study ${ }^{56}$.

XRD and AFM. The $\theta-2 \theta$ diffraction data measurements are done on a Bruker D8 Discover diffractometer $\mathrm{CuK}_{\alpha 1}(\lambda=1.54 \AA)$. Temperature-dependent electrical transport measurements are carried out in Physical Property Measurement system (Quantum Design) utilizing a four probe geometry. The surface morphology analysis of the various films are characterized on an atomic force microscope (AFM, Dimension V, Veeco).

THz-TDS. THz transmission spectra measurements of the $\mathrm{VO}_{2}$ films are carried out using the TeraView Spectra $3000 \mathrm{THz}$-TDS system, incorporated with a Janis ST100 -FTIR cryostat. The operational temperature range of the cryostat is $10 \mathrm{~K}$ to $450 \mathrm{~K}$. In this system low temperature-grown GaAs films is used for the generation and detection of $\mathrm{THz}$ waves. $\mathrm{THz}$ time-domain data of $\mathrm{VO}_{2}$ films are taken with respect to the signal transmitted through the bare substrate as a reference. Optical properties of the bare SLAO substrate are determined by THz-TDS, with vacuum as the reference signal. The complex refractive index, $\tilde{n}_{\text {sub }}$ of SLAO substrate is obtained to be $\tilde{n}_{\text {sub }} \approx 4.1-0.004 i$ and almost temperature and frequency-independent, and thus is a very suitable $\mathrm{THz}$ transparent material in our operational temperature and frequency range. The cryostat is equipped with a vertical motorized stage with a resolution of $2.5 \mu \mathrm{m}$ which is used to control the back and forth motion between the sample and reference positions.

The aperture diameter used is $7 \mathrm{~mm}$ which allowed for an accurate measurement of the THz signal for frequencies as low as $0.3 \mathrm{THz}$. For each sample and reference run, $900 \mathrm{THz}$ traces are taken in 3 minutes. The thickness difference between the film substrate and the bare substrate (reference), must be taken into account in our subsequent analysis ${ }^{57}$. The sample is kept in a high vacuum $\left(\sim 10^{-6} \mathrm{mbar}\right)$ for all our experiments.

1. Rao Popuri, S. et al. $\mathrm{VO}_{2}$ (A): Reinvestigation of crystal structure, phase transition and crystal growth mechanisms. J.Sol. St. Chem. 213, 79-86 (2014).

2. Wu, C., Wei, H., Ning, B. \& Xie, Y. New Vanadium Oxide Nanostructures: Controlled Synthesis and Their Smart Electrical Switching Properties. Adv. Mater. 22, 1972-1976 (2010).

3. Mattioli, G., Filippone, F., Alippi, P. \& Amore Bonapasta, A. Ab initio study of the electronic states induced by oxygen vacancies in rutile and anatase $\mathrm{TiO}_{2}$. Phys. Rev. B 78, 241201 (2008).

4. Mai, L. et al. Nanoscroll Buffered Hybrid Nanostructural $\mathrm{VO}_{2}(\mathrm{~B})$ Cathodes for High-Rate and Long-Life Lithium Storage. Adv. Mater. 25, 2969-2973 (2013).

5. Galy, J. A Proposal for (B) $\mathrm{VO}_{2}-(\mathrm{A}) \mathrm{VO}_{2}$ Phase Transition: A Simple Crystallographic Slip. J. Solid State Chem. 148, 224-228 (1999).

6. Katzke, H., Tolédano, P. \& Depmeier, W. Theory of morphotropic transformations in vanadium oxides. Phys. Rev. B 68, 024109 (2003).

7. Oka, Y., Yaob, T. \& Yamamoto, N. Structural Phase Transition of $\mathrm{VO}_{2}(\mathrm{~B})$ to $\mathrm{VO}_{2}$ (A). J. Mater. Chem. 1, 815-818 (1991).

8. Corr, S. A. et al. $\mathrm{VO}_{2}(\mathrm{~B})$ nanorods: solvothermal preparation, electrical properties, and conversion to rutile $\mathrm{VO}_{2}$ and $\mathrm{V}_{2} \mathrm{O}_{3}$. J. Mater. Chem. 19, 4362 (2009).

9. Zylbersztejn, A. Metal-insulator transition in vanadium dioxide. Phys. Rev. B 11, 4383-4395 (1975)

10. Oka, Y., Yao, T., Yamamoto, N., Ueda, Y. \& Hayashi, A. Phase Transition and $\mathrm{V}^{4+}$ $\mathrm{V}^{4+}$ pairing in $\mathrm{VO}_{2}(\mathrm{~B})$. J. Sol. St. Chem. 105, 271 (1993).

11. Leroux, C., Nihoul, G. \& Tendeloo, G. V. From $\mathrm{VO}_{2}(B)$ to $\mathrm{VO}_{2}(R)$ : Theoretical structures of $\mathrm{VO}_{2}$ polymorphs and in situ electron microscopy. Phys. Rev. B 57, $5111(1998)$.

12. Liu, J., Li, Q., Wang, T., Yu, D. \& Li, Y. Metastable Vanadium Dioxide Nanobelts: Hydrothermal Synthesis, Electrical Transport, and Magnetic Properties. Angew. Chem. 43, 5048-5052 (2004).

13. Imada, M., Fujimori, A. \& Tokura, Y. Metal-insulator transitions. Rev. Mod. Phys. 70, 1039-1263 (1998).

14. Goodenough, J. B. The Two Components of the Crystallographic Transition in $\mathrm{VO}_{2}$. J. Solid State Chem. 3, 490 (1971).

15. Nakano, M. et al. Collective bulk carrier delocalization driven by electrostatic surface charge accumulation. Nature 487, 459-462 (2012).

16. Jeong, J. et al. Suppression of Metal-Insulator Transition in $\mathrm{VO}_{2}$ by Electric FieldInduced Oxygen Vacancy Formation. Science 339, 1402-1405 (2013).
17. Okazaki, K. et al. Photoemission study of the metal-insulator transition in $\mathrm{VO}_{2} /$ $\mathrm{TiO}_{2}(001)$ : Evidence for strong electron-electron and electron-phonon interaction. Phys. Rev. B 69, 165104 (2004).

18. Liu, M. et al. Symmetry breaking and geometric confinement in $\mathrm{VO}_{2}$ : Results from a three-dimensional infrared nano-imaging. Appl. Phys. Lett. 104, 121905 (2014).

19. Ruzmetov, D., Senanayake, S. \& Ramanathan, S. X-ray absorption spectroscopy of vanadium dioxide thin films across the phase-transition boundary. Phys. Rev. B 75, 195102 (2007).

20. Koethe, T. C. et al. Transfer of Spectral Weight and Symmetry across the MetalInsulator Transition in $\mathrm{VO}_{2}$. Phys. Rev. Lett. 97, 116402 (2006).

21. Tao, Z. et al. Decoupling of Structural and Electronic Phase Transitions in $\mathrm{VO}_{2}$. Phys. Rev. Lett. 109, 166406 (2012).

22. Qazilbash, M. M. et al. Mott Transition in $\mathrm{VO}_{2}$ Revealed by Infrared Spectroscopy and Nano-Imaging. Science 318, 1750-1753 (2007).

23. Kim, H.-T. et al. Monoclinic and Correlated Metal Phase in $\mathrm{VO}_{2}$ as Evidence of the Mott Transition: Coherent Phonon Analysis. Phys. Rev. Lett. 97, 266401 (2006).

24. Cavalleri, A., Dekorsy, T., Chong, H., Kieffer, J. \& Schoenlein, R. Evidence for a structurally-driven insulator-to-metal transition in $\mathrm{VO}_{2}$ : A view from the ultrafast timescale. Phys. Rev. B 70, 161102 (2004).

25. Kübler, C. et al. Coherent Structural Dynamics and Electronic Correlations during an Ultrafast Insulator-to-Metal Phase Transition in $\mathrm{VO}_{2}$. Phys. Rev. Lett. 99, 116401 (2007).

26. Hilton, D. et al. Enhanced Photosusceptibility near $T_{c}$ for the Light-Induced Insulator-to-Metal Phase Transition in Vanadium Dioxide. Phys. Rev. Lett. 99 226401 (2007).

27. Jones, A. C., Berweger, S., Wei, J., Cobden, D. \& Raschke, M. B. Nano-optical Investigations of the Metal-Insulator Phase Behavior of Individual $\mathrm{VO}_{2}$ Microcrystals. Nano lett. 10, 1574-1581 (2010).

28. Quackenbush, N. F. et al. Nature of the Metal Insulator Transition in Ultrathin Epitaxial Vanadium Dioxide. Nano lett. 13, 4857-4861 (2013).

29. Jepsen, P. et al. Metal-insulator phase transition in a $\mathrm{VO}_{2}$ thin film observed with terahertz spectroscopy. Phys. Rev. B 74, 205103 (2006)

30. Cocker, T. L. et al. Terahertz conductivity of the metal-insulator transition in a nanogranular $\mathrm{VO}_{2}$ film. Appl. Phys. Lett. 97, 221905 (2010).

31. Mandal, P., Speck, A., Ko, C. \& Ramanathan, S. Terahertz spectroscopy studies on epitaxial vanadium dioxide thin films across the metal-insulator transition. Opt. Lett. 36, 1927 (2011)

32. Baxter, J. B. \& Schmuttenmaer, C. A. Carrier dynamics in bulk ZnO. I. Intrinsic conductivity measured by terahertz time-domain spectroscopy. Phys. Rev. B 80, 235205 (2009).

33. Baxter, J. B. \& Schmuttenmaer, C. A. Conductivity of ZnO Nanowires, Nanoparticles, and Thin Films Using Time-Resolved Terahertz Spectroscopy. J. Phys. Chem. B 110, 25229 (2006).

34. Tang, C. S. et al. Terahertz conductivity of topological surface states in $\mathrm{Bi}_{1.5} \mathrm{Sb}_{0.5} \mathrm{Te}_{1.8} \mathrm{Se}_{1.2}$. Sci. Rep. 3, 3513; DOI:10.1038/srep03513 (2014).

35. Lloyd-Hughes, J. \& Jeon, T.-I. A Review of the Terahertz Conductivity of Bulk and Nano-Materials. J. Infrared, Millimeter, Terahertz Waves 33, 871-925 (2012).

36. Zou, X. et al. Terahertz Conductivity of Twisted Bilayer Graphene. Phys. Rev. Lett. 110, 067401 (2013)

37. George, P. A. et al. Ultrafast Optical-Pump Terahertz-Probe on epitaxial graphene. Nano lett. 8, 4248-4251 (2008).

38. Valdés Aguilar, R. et al. Terahertz Response and Colossal Kerr Rotation from the Surface States of the Topological Insulator $\mathrm{Bi}_{2} \mathrm{Se}_{3}$. Phys. Rev. Lett. 108, 087403 (2012).

39. Bilbro, L. S. et al. Temporal correlations of superconductivity above the transition temperature in $\mathrm{La}_{2}-{ }_{\mathrm{x}} \mathrm{Sr}_{\mathrm{x}} \mathrm{CuO}_{4}$ probed by terahertz spectroscopy. Nature Phys. 7, 298-302 (2011)

40. Frenkel, A., Gao, F., Liu, Y., Whitaker, J. F. \& Uher, C. Conductivity peak, relaxation dynamics, and superconducting gap of $\mathrm{YBa}_{2} \mathrm{Cu}_{3} \mathrm{O}_{7}$ studied by terahertz and femtosecond optical spectroscopies. Phys. Rev. B 54, 1355 (1996).

41. Valdés Aguilar, R. et al. Pair-breaking effects and coherence peak in the terahertz conductivity of superconducting $\mathrm{BaFe}_{2}-{ }_{2 \mathrm{x}} \mathrm{Co}_{2 \mathrm{x}} \mathrm{As}_{2}$ thin films. Phys. Rev. B 82, 180514 (2010).

42. Ulbricht, R., Hendry, E., Shan, J., Heinz, T. F. \& Bonn, M. Carrier dynamics in semiconductors studied with time-resolved terahertz spectroscopy. Rev. Mod. Phys. 83, 543-586 (2011).

43. Walther, M. et al. Terahertz conductivity of thin gold films at the metal-insulator percolation transition. Phys. Rev. B 76, 125408 (2007).

44. Duvillaret, L., Garet, F. \& Coutaz, J. L. A Reliable Method for Extraction of Material Parameters in Terahertz Time-Domain Spectroscopy. IEEE J. Sel. Top. Quantum Electron. 2, 739 (1996).

45. Barker, A., Verleur, H. \& Guggenheim, H. Infrared Optical Properties of Vanadium Dioxide Above and Below the Transition Temperature. Phys. Rev. Lett. 17, 1286-1289 (1966)

46. Choi, H. S., Ahn, J. S., Jung, J. H., Noh, T. W. \& Kim, D. H. Mid-infrared properties of a $\mathrm{VO}_{2}$ film near the metal-insulator transition. Phys. Rev. B 54, 4621 (1996).

47. Smith, N. Classical generalization of the Drude formula for the optical conductivity. Phys. Rev. B 64, 155106 (2001).

48. Turner, G. M., Beard, M. C. \& Schmuttenmaer, C. A. Carrier Localization and Cooling in Dye-Sensitized Nanocrystalline Titanium Dioxide. J. Phys. Chem. B 106, 11716-11719 (2002) 
49. Qazilbash, M. et al. Infrared spectroscopy and nano-imaging of the insulator-tometal transition in vanadium dioxide. Phys. Rev. B 79, 075107 (2009).

50. Cooke, D. et al. Transient terahertz conductivity in photoexcited silicon nanocrystal films. Phys. Rev. B 73, 193311 (2006).

51. Němec, H., Kužel, P. \& Sundström, V. Far-infrared response of free charge carriers localized in semiconductor nanoparticles. Phys. Rev. B 79, 115309 (2009).

52. Zou, X. et al. Temperature-dependent terahertz conductivity of tin oxide nanowire films. J. Phys. D: Appl. Phys. 45, 465101 (2012).

53. Ruzmetov, D., Heiman, D., Claflin, B., Narayanamurti, V. \& Ramanathan, S. Hall carrier density and magnetoresistance measurements in thin-film vanadium dioxide across the metal-insulator transition. Phys. Rev. B 79, 153107 (2009).

54. Qazilbash, M. et al. Correlated metallic state of vanadium dioxide. Phys. Rev. B 74, 205118 (2006).

55. Karaoglan-Bebek, G., Hoque, M. N. F., Holtz, M., Fan, Z. \& Bernussi, A. A. Continuous tuning of $\mathrm{W}$-doped $\mathrm{VO} 2$ optical properties for terahertz analog applications. Appl. Phys. Lett. 105, 201902 (2014).

56. Srivastava, A. et al. Selective Growth of Single Phase $\mathrm{VO}_{2}(\mathrm{~A}, \mathrm{~B}$ and $\mathrm{M})$ Polymorph Thin Films. Appl. Phys. Lett. Mater.3, 026101 (2015).

57. Kadlec, C. et al. Temperature and electric field tuning of the ferroelectric soft mode in a strained $\mathrm{SrTiO}_{3} / \mathrm{DyScO}_{3}$ heterostructure. Phys. Rev. B 80, 174116 (2009)

\section{Acknowledgments}

We thank the NUSNNI-NanoCore at the National University of Singapore, Singapore and National Research Foundation (NRF), Singapore, under the Competitive Research Program (CRP) "Tailoring Oxide Electronics by Atomic Control” NRF2008NRF-CRP002-024, CRP "Interface Science and Technology" NRF-CRP4-2008-04, National University of Singapore (NUS) cross-faculty grant and FRC (ARF Grant No. R-144-000- 278-112), MOE Tier 1
RG13/12 and MOE2014-T1-001-056 for the financial support. We also acknowledge the funding support from the Singapore NRF through the Singapore-Berkeley Research Initiative for Sustainable Energy (SinBeRISE) CREATE Programme.

\section{Author contributions}

T.V. and E.E.M.C. conceived the project. J.L., T.V. and E.E.M.C. designed the research and E.E.M.C. supervised the project. A.S. fabricated the samples and conducted electrical measurements. H.R. took X.R.D. measurements. J.L. performed the AFM measurements. THz-TDS data were taken by J.L. and C.L. and analyzed by J.L. and E.E.M.C. The manuscript was prepared by J.L. and E.E.M.C. with assistance from T.V., C.L. and A.S. All the authors contributed to discussion on the results for the manuscript.

\section{Additional information}

Supplementary information accompanies this paper at http://www.nature.com/ scientificreports

Competing financial interests: The authors declare no competing financial interests.

How to cite this article: Lourembam, J. et al. New Insights into the Diverse Electronic Phases of a Novel Vanadium Dioxide Polymorph: A Terahertz Spectroscopy Study. Sci. Rep. 5, 9182; DOI:10.1038/srep09182 (2015).

This work is licensed under a Creative Commons Attribution 4.0 International License. The images or other third party material in this article are included in the article's Creative Commons license, unless indicated otherwise in the credit line; if the material is not included under the Creative Commons license, users will need to obtain permission from the license holder in order to reproduce the material. To view a copy of this license, visit http://creativecommons.org/licenses/by/4.0/ 